\title{
Higher-Order Families of Multiple Root Finding Methods Suitable for Non-Convergent Cases and their Dynamics
}

\author{
Ramandeep Behl ${ }^{a}$, Vinay $\mathrm{Kanwar}^{b}$ and Young Ik Kim \\ ${ }^{a}$ King Abdulaziz University \\ Department of Mathematics, Jeddah 21589, Saudi Arabia \\ ${ }^{b}$ Panjab University \\ University Institute of Engineering and Technology, Chandigarh-160 014, \\ India \\ ${ }^{c}$ Dankook University \\ Department of Applied Mathematics, Cheonan 330-714, South Korea \\ E-mail: ramanbeh187@yahoo.in \\ E-mail: vmithil@yahoo.co.in \\ E-mail(corresp.): yikbell@yahoo.co.kr
}

Received September 24, 2018; revised May 8, 2019; accepted May 9, 2019

\begin{abstract}
In this paper, we present many new one-parameter families of classical Rall's method (modified Newton's method), Schröder's method, Halley's method and super-Halley method for the first time which will converge even though the guess is far away from the desired root or the derivative is small in the vicinity of the root and have the same error equations as those of their original methods respectively, for multiple roots. Further, we also propose an optimal family of iterative methods of fourth-order convergence and converging to a required root in a stable manner without divergence, oscillation or jumping problems. All the methods considered here are found to be more effective than the similar robust methods available in the literature. In their dynamical study, it has been observed that the proposed methods have equal or better stability and robustness as compared to the other methods.
\end{abstract}

Keywords: multiple roots, Rall's method, Schröder's method, super-Halley's method, basins of attraction.

AMS Subject Classification: 41A25; $65 \mathrm{H} 05$.

Copyright (c) 2019 The Author(s). Published by VGTU Press

This is an Open Access article distributed under the terms of the Creative Commons Attribution License (http://creativecommons.org/licenses/by/4.0/), which permits unrestricted use, distribution, and reproduction in any medium, provided the original author and source are credited. 


\section{Introduction}

One topic which has always been of paramount importance in computational mathematics is that of approximating efficiently multiple roots of nonlinear equations of the form

$$
f(x)=0
$$

where $f: I \subseteq \mathbb{R} \rightarrow \mathbb{R}$ is a nonlinear sufficiently differentiable function in an interval $I$.

To solve nonlinear equation (1.1), one can use classical iterative methods such as Rall's method (modified Newton's method) [12], [11], Schröder's method [13], Halley's and super-Halley method [5]. Perhaps, the most celebrated of all such iterative methods is the classical Rall's method (also known as modified Newton's method) for finding multiple roots of nonlinear equation (1.1), given by

$$
x_{n+1}=x_{n}-m \frac{f\left(x_{n}\right)}{f^{\prime}\left(x_{n}\right)}, \quad n \geq 0 .
$$

It converges quadratically and requires the prior knowledge of multiplicity $m$.

However, as it is well-known that the prominent one-point modified Newton's method has some drawbacks. This method may be sensitive to the quality of the initial guess. Moreover, the iteration can be aborted due to overflow or leads to divergence, if the derivative of a function at an iterative point is singular or almost singular $\left(f^{\prime}\left(x_{n}\right)=0\right)$, which restricts their practical applications. Therefore, more effective globally convergent algorithms are still needed.

In order to overcome these problems, Kanwar et al. [4] proposed the following modification over Rall's method as

$$
x_{n+1}=x_{n}-m \frac{f\left(x_{n}\right)}{f^{\prime}\left(x_{n}\right)-\gamma f\left(x_{n}\right)}, \gamma \in \mathbb{R} .
$$

It satisfies the following error equation

$$
e_{n+1}=\frac{\left(c_{1}-\gamma\right)}{m} e_{n}^{2}+O\left(e_{n}^{3}\right)
$$

$c_{k}=\frac{m !}{(m+k) !} \frac{f^{(m+k)}\left(r_{m}\right)}{f^{(m)}\left(r_{m}\right)}, k=1,2,3, \ldots$ and $r_{m}$ is a multiple root which is different from Rall's method. This family converges quadratically under the condition that $f^{\prime}\left(x_{n}\right)-\gamma f\left(x_{n}\right) \neq 0$, while $f^{\prime}\left(x_{n}\right)=0$ is permitted at some points. This technique provides an alternative to the failure situation of existing classical Rall's method. Unfortunately, this technique does not have the same error equation as that of existing classical Rall's method.

Therefore, we intend to develop a scheme that will converge to the required root even though the guess is far away from the required root or derivative is very small in the vicinity of the required root and also has the same error equation as that of original Rall's method. Further, we present many new highly efficient families of Schröder's method, super-Halley method and Halley's method respectively which will not only converge to the required root but also have the same error equations as those of existing classical methods. 
Furthermore, a new fourth-order optimal family of methods has been developed by discretization of the second-order derivative involved in the family of super-Halley method.

\section{Development of iterative schemes}

Let us consider a curve in the following form

$$
y=a_{1}\left(x-x_{n}\right)+a_{2} e^{\gamma\left(x-x_{n}\right)},
$$

where $\gamma \in \mathbb{R}, a_{1}$ and $a_{2}$ are arbitrary constants to be determined. To be osculating, we require

$$
y\left(x_{n}\right)=f\left(x_{n}\right), y^{\prime}\left(x_{n}\right)=f^{\prime}\left(x_{n}\right),
$$

which lead to

$$
a_{1}=f^{\prime}\left(x_{n}\right)-\gamma f\left(x_{n}\right) \text { and } a_{2}=f\left(x_{n}\right) .
$$

Suppose the curve $(2.1)$ cuts the $x$-axis at $x_{n+1}$, then $y\left(x_{n+1}\right)=0$ and it follows from (2.1) that

$$
a_{1}\left(x_{n+1}-x_{n}\right)+a_{2} e^{\gamma\left(x_{n+1}-x_{n}\right)}=0 .
$$

From (2.2), we get

$$
x_{n+1}=x_{n}-\frac{f\left(x_{n}\right)}{f^{\prime}\left(x_{n}\right)-\gamma f\left(x_{n}\right)} e^{\gamma\left(x_{n+1}-x_{n}\right)} .
$$

Approximating $\left(x_{n+1}-x_{n}\right)$ on the right-hand side of equation (2.3) by the correction factor: $-\frac{f\left(x_{n}\right)}{f^{\prime}\left(x_{n}\right)-\gamma f\left(x_{n}\right)}$ (given in formula (1.2) for $m=1$ ), one gets

$$
x_{n+1}=x_{n}-\frac{f\left(x_{n}\right)}{f^{\prime}\left(x_{n}\right)-\gamma f\left(x_{n}\right)} e^{\frac{-\gamma f\left(x_{n}\right)}{f^{\prime}\left(x_{n}\right)-\gamma f\left(x_{n}\right)}} .
$$

The error equation of scheme (2.4) is given by

$$
e_{n+1}=c_{1} e_{n}^{2}+O\left(e_{n}^{3}\right) \text {. }
$$

This is a new one-parameter family of Newton's method. The beauty of this family is that it has the same error equation as Newton's method. In addition, scheme (2.4) does not fail even the guess is far away from the required root or become very small in the vicinity of the required root. Now, we want to extend this idea for multiple roots. Consider the following modification to the above mentioned one-point iterative family (2.4)

$$
x_{n+1}=x_{n}-m \frac{f\left(x_{n}\right)}{f^{\prime}\left(x_{n}\right)-\gamma f\left(x_{n}\right)} e^{\frac{-\gamma f\left(x_{n}\right)}{f^{\prime}\left(x_{n}\right)-\gamma f\left(x_{n}\right)}} .
$$

Further, we want to simplify the body structure of the above family and also introduce some higher-order variants of this family (2.5). Therefore, we consider $\left|\frac{\gamma f\left(x_{n}\right)}{f^{\prime}\left(x_{n}\right)-\gamma f\left(x_{n}\right)}\right|<<1$ and using Taylor series expansion for $e^{\frac{-\gamma f\left(x_{n}\right)}{f^{\prime}\left(x_{n}\right)-\gamma f\left(x_{n}\right)}}$, we obtain

$$
e^{\frac{-\gamma f\left(x_{n}\right)}{f^{\prime}\left(x_{n}\right)-\gamma f\left(x_{n}\right)}} \approx 1-\frac{\gamma f\left(x_{n}\right)}{f^{\prime}\left(x_{n}\right)-\gamma f\left(x_{n}\right)} .
$$


Using this approximate value of $\mu_{n}=e^{\frac{-\gamma f\left(x_{n}\right)}{f^{\prime}\left(x_{n}\right)-\gamma f\left(x_{n}\right)}}$ in formula (2.5), one gets

$$
x_{n+1}=x_{n}-m \frac{f\left(x_{n}\right)\left(f^{\prime}\left(x_{n}\right)-2 \gamma f\left(x_{n}\right)\right)}{\left(f^{\prime}\left(x_{n}\right)-\gamma f\left(x_{n}\right)\right)^{2}} .
$$

This is another new one-parameter family of Newton's method. Let us assume that the corrector factor as $g(x)=\frac{m f\left(x_{n}\right)}{f^{\prime}\left(x_{n}\right)-\gamma f\left(x_{n}\right)} \mu_{n}$, then we have

$$
g^{\prime}(x)=m\left(\frac{f^{\prime}\left(x_{n}\right)-2 \gamma f\left(x_{n}\right)}{f^{\prime}\left(x_{n}\right)-\gamma f\left(x_{n}\right)}\right)\left(\frac{f^{\prime}\left(x_{n}\right)^{2}-f\left(x_{n}\right) f^{\prime \prime}\left(x_{n}\right)}{\left(f^{\prime}\left(x_{n}\right)-\gamma f\left(x_{n}\right)\right)^{2}}\right) e^{\frac{-\gamma f\left(x_{n}\right)}{f^{\prime}\left(x_{n}\right)-\gamma f\left(x_{n}\right)}}
$$

Now, by applying Newton's method to the correction factor $\frac{m f\left(x_{n}\right)}{f^{\prime}\left(x_{n}\right)-\gamma f\left(x_{n}\right)} \mu_{n}$ of formula (2.5), one gets

$$
x_{n+1}=x_{n}-\frac{f\left(x_{n}\right)\left(f^{\prime}\left(x_{n}\right)-\gamma f\left(x_{n}\right)\right)^{2}}{\left(f^{\prime}\left(x_{n}\right)^{2}-f\left(x_{n}\right) f^{\prime \prime}\left(x_{n}\right)\right)\left(f^{\prime}\left(x_{n}\right)-2 \gamma f\left(x_{n}\right)\right)} .
$$

This is a one-parameter modified family of Schröder's method [13] for an equation having multiple roots of multiplicity $m \geq 1$ unknown. It is easy to verify that this method has quadratic convergence. The order of convergence of family (2.6) is analyzed in Theorem 1.

Further, from formula (2.6) and (2.7), one gets

$$
\begin{aligned}
x_{n+1}= & x_{n}-\frac{1}{2}\left[\frac{m f\left(x_{n}\right)\left(f^{\prime}\left(x_{n}\right)-2 \gamma f\left(x_{n}\right)\right)}{\left(f^{\prime}\left(x_{n}\right)-\gamma f\left(x_{n}\right)\right)^{2}}\right. \\
& \left.+\frac{f\left(x_{n}\right)\left(f^{\prime}\left(x_{n}\right)-\gamma f\left(x_{n}\right)\right)^{2}}{\left(f^{\prime}\left(x_{n}\right)^{2}-f\left(x_{n}\right) f^{\prime \prime}\left(x_{n}\right)\right)\left(f^{\prime}\left(x_{n}\right)-2 \gamma f\left(x_{n}\right)\right)}\right] .
\end{aligned}
$$

Note that this is a new family of famous cubically convergent super-Halley method and can be viewed as an arithmetic mean of two functions namely, $\frac{m f\left(x_{n}\right)\left(f^{\prime}\left(x_{n}\right)-2 \gamma f\left(x_{n}\right)\right)}{\left(f^{\prime}\left(x_{n}\right)-\gamma f\left(x_{n}\right)\right)^{2}}$ and $\frac{f\left(x_{n}\right)\left(f^{\prime}\left(x_{n}\right)-\gamma f\left(x_{n}\right)\right)^{2}}{\left(f^{\prime}\left(x_{n}\right)^{2}-f\left(x_{n}\right) f^{\prime \prime}\left(x_{n}\right)\right)\left(f^{\prime}\left(x_{n}\right)-2 \gamma f\left(x_{n}\right)\right)}$.

If we take the harmonic mean in (2.8) instead of the arithmetic one, we get

$$
\begin{aligned}
& x_{n+1}=x_{n} \\
& -\frac{2 m f\left(x_{n}\right)\left(f^{\prime}\left(x_{n}\right)-2 \gamma f\left(x_{n}\right)\right)\left(f^{\prime}\left(x_{n}\right)-\gamma f\left(x_{n}\right)\right)^{2}}{m\left(f^{\prime}\left(x_{n}\right)-2 \gamma f\left(x_{n}\right)\right)^{2}\left(f^{\prime}\left(x_{n}\right)^{2}-f\left(x_{n}\right) f^{\prime \prime}\left(x_{n}\right)\right)+\left(f^{\prime}\left(x_{n}\right)-\gamma f\left(x_{n}\right)\right)^{4}} .
\end{aligned}
$$

This is a modification over the well-known cubically convergent Halley's method. The first most striking feature of this contribution is that we have developed families of Rall's, Schröder's, super-Halley and Halley's methods for the first time which will converge even though the guess is far from the desired root or the derivative is small in the vicinity of the root and have the same error equations as those of their original methods respectively. It is also interesting to note that for $\gamma=0$, these formulas reduce to Rall's [12], Schröder's [13], super-Halley and Halley's [5] methods for multiple roots respectively. 


\section{Optimal families of multi-point methods}

The main practical difficulty associated with the recently developed methods is that they require lengthy computation of second-order derivatives that reduce the efficiency and accuracy of the methods. Therefore, second-order derivative free methods are still needed. In the past and recent years, many multi-point iterative methods have been proposed for solving nonlinear equations that improve local convergence order of the classical modified Newton's method (Rall's method), see [8], [7], [15], [19], [16] and the references cited therein.

Therefore, obtaining new optimal methods of fourth order, not requiring the computation of second-order derivative, is a very important and interesting task from a practical point of view, because their corresponding efficiency index [10] is 1.587. Motivated in this direction, we develop many new interesting fourth-order optimal families of Jarratt's type methods free from second-order derivatives.

Let us consider a Newton-like iterate $w_{n}=x_{n}-\frac{2 m}{m+2} v\left(x_{n}\right)$, where $v \equiv v\left(x_{n}\right)=\frac{f\left(x_{n}\right)\left\{f^{\prime}\left(x_{n}\right)-2 \gamma f\left(x_{n}\right)\right\}}{\left\{f^{\prime}\left(x_{n}\right)-\gamma f\left(x_{n}\right)\right\}^{2}}$ and expand $f^{\prime}\left(x_{n}-\frac{2 m}{m+2} v\right)$ about a point $x=x_{n}$ by Taylor's series expansion as follows:

$$
f^{\prime}\left(w_{n}\right)=f^{\prime}\left(x_{n}\right)-\frac{2 m}{m+2} v f^{\prime \prime}\left(x_{n}\right)+O\left(\frac{2 m}{m+2} v\right)^{2} .
$$

Therefore, one obtains another approximation for $f^{\prime \prime}\left(x_{n}\right)$ as follows:

$$
f^{\prime \prime}\left(x_{n}\right) \approx \frac{f^{\prime}\left(x_{n}\right)-f^{\prime}\left(w_{n}\right)}{2 m v /(m+2)},
$$

where $v$ is as defined earlier. Using this approximation of $f^{\prime \prime}\left(x_{n}\right)$ in the expression (2.8), we have

$$
\begin{gathered}
x_{n+1}=x_{n}-\frac{1}{2}\left[2 m v f\left(x_{n}\right)\left(f^{\prime}\left(x_{n}\right)-\gamma f\left(x_{n}\right)\right)^{2} /\left(( f ^ { \prime } ( x _ { n } ) - 2 \gamma f ( x _ { n } ) ) \left(2 m v f^{\prime}\left(x_{n}\right)^{2}\right.\right.\right. \\
\left.\left.\left.-(m+2) f\left(x_{n}\right)\left(f^{\prime}\left(x_{n}\right)-f^{\prime}\left(w_{n}\right)\right)\right)\right)+\frac{m f\left(x_{n}\right)\left(f^{\prime}\left(x_{n}\right)-2 \gamma f\left(x_{n}\right)\right)}{\left(f^{\prime}\left(x_{n}\right)-\gamma f\left(x_{n}\right)\right)^{2}}\right] .
\end{gathered}
$$

We introduce some disposable parameters and after simplifications, we obtain

$$
\begin{aligned}
x_{n+1}= & x_{n} \\
& -\frac{m f\left(x_{n}\right)\left[2 v a_{5} \lambda_{1}^{4}+\left(2 a_{6} m v f^{\prime}\left(x_{n}\right)^{2}+a_{7}(m+2) f\left(x_{n}\right) \beta\right) \lambda_{2}^{2}\right]}{2\left((m+2) f\left(x_{n}\right) \beta+2 a_{8} m v f^{\prime}\left(x_{n}\right)^{2}\right) \lambda_{2} \lambda_{1}^{2}},
\end{aligned}
$$

where $\lambda_{1}=f^{\prime}\left(x_{n}\right)-\gamma f\left(x_{n}\right), \lambda_{2}=f^{\prime}\left(x_{n}\right)-2 \gamma f\left(x_{n}\right), \beta=f^{\prime}\left(w_{n}\right)-f^{\prime}\left(x_{n}\right)$, while $a_{5}, a_{6}, a_{7}$ and $a_{8}$ are disposable parameters such that the order of convergence reaches at the optimal (according to Kung-Traub conjecture [6]) level four without using any more functional evaluations. Theorem 1 indicates that under what choices on the disposable parameters in (3.1), the order of convergence will reach at the optimal level four. 


\section{Convergence analysis}

Theorem 1. Let $f: D \subseteq \mathbb{R} \rightarrow \mathbb{R}$ be a sufficiently smooth function defined on an open interval $D$, enclosing a multiple zero of $f(x)$, say $x=r_{m}$ with multiplicity $m \geq 1$. Then, for $\gamma \in \mathbb{R}$, iteration schemes defined by formulas:

(i) Schemes (2.5) and (2.7) have quadratic order of convergence.

(ii) Schemes (2.8) and (2.9) have cubic order of convergence.

(iii) Scheme (3.1) has optimal fourth-order of convergence if

$$
\left\{\begin{array}{l}
a_{5}=\frac{1}{4}(2+m)(4 \mu+m(3 \mu-1)), \\
a_{6}=-\frac{(2+m)(-4+m+4 \mu+m \mu)}{4 m}, \\
a_{7}=-(m-2), \quad a_{8}=-(2+m)(-1+\mu) /(2 m),
\end{array}\right.
$$

respectively, where $\mu=\left(\frac{m}{2+m}\right)^{m}$.

Proof. Let $x=r_{m}$ be a multiple zero of $f(x)$ and $e_{n}=x_{n}-r_{m}$ be the error at the $n$-th iteration. Expanding $f\left(x_{n}\right)$ and $f^{\prime}\left(x_{n}\right)$ about $x=r_{m}$ by the Taylor's series expansion (with the help of computer algebra software Mathematica 9), we have

$$
\begin{aligned}
f\left(x_{n}\right)= & \frac{1}{m !} f^{(m)}\left(r_{m}\right) e_{n}^{m}\left[1+e_{n} c_{1}+e_{n}^{2} c_{2}+e_{n}^{3} c_{3}+e_{n}^{4} c_{4}+O\left(e_{n}^{5}\right)\right] \\
f^{\prime}\left(x_{n}\right)= & \frac{1}{m !} f^{(m)}\left(r_{m}\right) e_{n}^{m-1}\left[m+(m+1) e_{n} c_{1}+(m+2) e_{n}^{2} c_{2}\right. \\
& \left.+(m+3) e_{n}^{3} c_{3}+(m+4) e_{n}^{4} c_{4}+O\left(e_{n}^{5}\right)\right] \\
f^{\prime \prime}\left(x_{n}\right)= & \frac{1}{m !} f^{(m)}\left(r_{m}\right) e_{n}^{m-2}\left[-m+m^{2}+m(1+m) c_{1} e_{n}\right. \\
& \left.+\left(2+3 m+m^{2}\right) c_{2} e_{n}^{2}+\left(6+5 m+m^{2}\right) c_{3} e_{n}^{3}+O\left(e_{n}^{4}\right)\right]
\end{aligned}
$$

respectively. Making use of equations (4.2)-(4.4) in iterative schemes (2.5) and (2.7) and after some simplifications, one can have the following error equations

$$
\begin{aligned}
& e_{n+1}=\frac{c_{1}}{m} e_{n}^{2}+O\left(e_{n}^{3}\right), \quad \text { (same as Rall's method) } \\
& e_{n+1}=-\frac{c_{1}}{m} e_{n}^{2}+O\left(e_{n}^{3}\right), \quad \text { (same as Schröder's method) }
\end{aligned}
$$

respectively. This proves the quadratic convergence of iterative schemes (2.5) and (2.7).

Further, making use of equations (4.2)-(4.4) in iterative schemes (2.8) and (2.9) and after some simplifications, one can have the following error equations

$$
\begin{aligned}
& e_{n+1}=\left(\frac{(m-1) c_{1}^{2}-2 m c_{2}}{2 m^{2}}\right) e_{n}^{3}+O\left(e_{n}^{4}\right), \quad \text { (same as super-Halley method), } \\
& e_{n+1}=\left(\frac{(m+1) c_{1}^{2}-2 m c_{2}}{2 m^{2}}\right) e_{n}^{3}+O\left(e_{n}^{4}\right), \quad \text { (same as Halley's method), }
\end{aligned}
$$


respectively. This proves the cubic convergence of iterative schemes (2.8) and (2.9).

Using equations (4.2) and (4.3), one gets

$$
\begin{aligned}
v=\frac{f\left(x_{n}\right)\left(f^{\prime}\left(x_{n}\right)-2 \gamma f\left(x_{n}\right)\right)}{\left(f^{\prime}\left(x_{n}\right)-\gamma f\left(x_{n}\right)\right)^{2}}= & \frac{e_{n}}{m}-\frac{c_{1} e_{n}^{2}}{m^{2}} \\
& +\frac{\left((1+m) c_{1}^{2}-2 m c_{2}-\gamma^{2}\right) e_{n}^{3}}{m^{3}}+O\left(e_{n}^{4}\right),
\end{aligned}
$$

and

$$
\begin{gathered}
f^{\prime}\left(x_{n}-\frac{2 m}{m+2} v\right)=f^{(m)}\left(r_{m}\right) e_{n}^{m-1}\left[\frac{\mu(2+m)}{m !}+\frac{\mu\left(2 m+3 m^{2}+m^{3}-4\right) c_{1} e_{n}}{m^{2} m !}\right. \\
\left.\quad+\frac{\mu\left(4(2-m) c_{1}^{2}+m\left(2\left(m+m^{2}-2\right) \gamma^{2}+b_{0} c_{2}\right)\right) e_{n}^{2}}{m^{4} m !}+O\left(e_{n}^{3}\right)\right]
\end{gathered}
$$

where $b_{0}=m\left(4 m+4 m^{2}+m^{3}-8\right)$. Substituting (4.1)-(4.3) and (4.5) in iterative scheme (3.1) and after some simplification, one can have the following error equation

$$
e_{n+1}=\frac{\left[2 \mu b_{1} c_{1}^{3}-3 m^{2}(2+m)^{2} c_{1}\left((1-\mu) \gamma^{2}+2 m \mu c_{2}\right)+6 m^{5} \mu c_{3}\right] e_{n}^{4}}{6 \mu m^{4}(2+m)^{2}}+O\left(e_{n}^{5}\right),
$$

where $b_{1}=(2+m)^{2}\left(2 m+2 m^{2}+m^{3}-2\right)$. This completes the proof of the Theorem 1. It is noteworthy that all our methods are also working for simple roots if you simply taking the value of $m=1$ with same order.

\section{Extraneous fixed points}

Multipoint iterative methods [6] solving a generic nonlinear equation of the form $f(x)=0$ can be represented by a discrete dynamical system

$$
x_{n+1}=R_{f}\left(x_{n}\right),
$$

where $R_{f}$ is the iteration function whose fixed points are zeros of $f(x)$ under consideration. The iteration function $R_{f}$, however, might possess other fixed points that are not zeros of $f$. Such fixed points different from zeros of $f$ are called the extraneous fixed points $[3,17]$ of the iteration function $R_{f}$. Extraneous fixed points may form attractive, indifferent, repulsive cycles or periodic orbits to display chaotic dynamics behind the basin of attraction under investigation. The existence of such extraneous fixed points would affect the global iteration dynamics, which was demonstrated via König functions by Vrscay and Gilbert [17]. Especially the presence of attractive cycles induced by the extraneous fixed points of $R_{f}$ may alter the basin of attractions due to the trapped sequence $\left\{x_{n}\right\}$. Even in the case of repulsive or indifferent fixed points, an initial value $x_{0}$ chosen near a desired root may converge to another unwanted remote root. Indeed, these aspects of the Schröder functions [17] were observed 
in an application to the family of functions $\left\{f_{k}(x)=x^{k}-1, k \geq 2\right\}$. Such dynamical aspects motivate our investigation of the extraneous fixed points that may affect the basins of attraction for the proposed methods (3.1).

For notational convenience, we first denote $h=\frac{f\left(x_{n}\right)}{f^{\prime}\left(x_{n}\right)}$ and $t=\frac{f^{\prime}\left(w_{n}\right)}{f^{\prime}\left(x_{n}\right)}$. Then with $v=\frac{h(1-2 \gamma h)}{(1-\gamma h)^{2}}$, iterative methods (3.1) can be written as

$$
\begin{aligned}
w_{n} & =x_{n}-\frac{2 m}{m+2} v\left(x_{n}\right), \\
x_{n+1} & =x_{n}-h \frac{m}{2}\left[\frac{\left(a_{7}(t-1)(m+2) h+2 a_{6} m v\right)(1-2 h \gamma)^{2}+2 a_{5} v(1-h \gamma)^{4}}{\left((t-1)(m+2) h+2 a_{8} m v\right)(1-2 h \gamma)(1-h \gamma)^{2}}\right] .
\end{aligned}
$$

The above equation can be represented in the form of a weighted Newtonian discrete dynamical system:

$$
x_{n+1}=R_{f}\left(x_{n}\right)=x_{n}-\frac{f\left(x_{n}\right)}{f^{\prime}\left(x_{n}\right)} H_{f}\left(x_{n}\right),
$$

where $H_{f}\left(x_{n}\right)=\frac{m}{2} \frac{\left(a_{7}(t-1)(m+2) h+2 a_{6} m v\right)(1-2 h \gamma)^{2}+2 a_{5} v(1-h \gamma)^{4}}{\left((t-1)(m+2) h+2 a_{8} m v\right)(1-2 h \gamma)(1-h \gamma)^{2}}$ with coefficients $a_{5}, a_{6}, a_{7}, a_{8}$ given by (4.1). It is clearly the form of $H_{f}\left(x_{n}\right)$ to characterize a variety of iterative methods. The zero $r_{m}$ of $f(x)$ is obviously a fixed point of $R_{f}$. The points $\xi \neq r_{m}$ for which $H_{f}(\xi)=0$ are extraneous fixed points of $R_{f}$.

Let $H(z)$ represent a $H_{f}(z)$ when $f(z)$ is a finite-order rational function of $z$. Then it would be of great interest for us to investigate the complex dynamics of the rational iterative map $R_{p}$ of the form

$$
z_{n+1}=R_{p}\left(z_{n}\right)=z_{n}-\frac{p\left(z_{n}\right)}{p^{\prime}\left(z_{n}\right)} H\left(z_{n}\right),
$$

in connection with the basins of attraction for a variety of polynomials $p\left(z_{n}\right)$. Clearly, $R_{p}(z)$ represents the classical Newton's method with weighing function $H(z)$ and may possess its fixed points as zeros of $p(z)$ or extraneous fixed points associated with $H(z)$.

Indeed, if we take $f(z)=p(z)$, the same polynomial as given in (5.1), then we get the usual rational iterative map $\mathcal{R}_{p}$ of the form

$$
z_{n+1}=\mathcal{R}_{p}\left(z_{n}\right)=z_{n}-\frac{p\left(z_{n}\right)}{p^{\prime}\left(z_{n}\right)} \mathcal{H}\left(z_{n}\right)
$$

The complex dynamics of (5.2) along with its basins of attraction will be described later in the first part of Section 7.

We now turn to a different dynamics originated from the extraneous fixed points of iterative map (5.1). We are interested also in the investigation of unified dynamics associated with these extraneous fixed points. To this end, we apply a simple quadratic polynomial raised to the power of multiplicity $m$, i.e., $f(z)=\left(z^{2}-1\right)^{m}$ to $H_{f}\left(x_{n}\right)$, simple-root cases of which were introduced by Cayley [2] and Vrscay et al. [17] in dynamical studies of the Schröder and 
König functions for a family of functions $f_{k}(z)=z^{k}-1, k \in \mathbb{N}$ to minimize perturbations of the Julia set boundaries.

Hence in this section we will exclusively discuss the complex dynamics of (5.1) associated with its extraneous fixed points. To this end, we first choose $\gamma=1$ and denote (3.1) by $O M_{4}$ to solve a variety of nonlinear equations which are mentioned in the following examples $1-3$. We write $H(z)$ in the form of

$$
H(z)=A(z) \cdot \frac{F(z)}{D(z)}
$$

where $A(z)$ is a function of $z$ whose roots may contain $z=0$ (which causes infinity to $h$ ) or $z= \pm 1$ (which satisfy $\left(z^{2}-1\right)^{m}$ ) independently of the extraneous fixed points of $H ; F(z)$ and $D(z)$ are polynomials having no common factors; $F(z)$ may indeed contain the extraneous fixed points of $H$.

In order to compare the dynamics behavior of Method $O M_{4}$ related to extraneous fixed points, let us now employ the existing four optimal methods with quartic convergence. We conveniently denote these existing optimal fourth-order methods by $S M_{4}, Z M_{4}, S S M_{4}$ and $L M_{4}$, which are proposed by Soleymani et al. [16] with their best expression (18), proposed by Zhou et al. [19] with their expression (11), proposed by Sharma and Sharma [15], and proposed by Li et al. [8] with their expression (75), respectively.

By similarly following the development procedure of $H(z)$ for Method $\mathrm{OM}_{4}$, we find the corresponding $H(z)$ for existing methods $L M_{4}, S S M_{4}, Z M_{4}, S M_{4}$ as follows:

$$
H(z)= \begin{cases}\frac{\left(4-m^{2}\right) \delta \sigma^{m-1}+m \lambda}{(2+m) \delta \sigma^{m-1}-\lambda}, & \text { for } L M_{4}, \\ \frac{(m+2) \rho^{2}-2(m-1)(m+2) \delta \rho \sigma^{m-1}+\left(8-4 m+m^{3}\right) \delta^{2} \sigma^{2 m-2}}{\delta^{2} \sigma^{2 m-2}}, & \text { for } S S M_{4}, \\ \frac{\left(m^{3}+6 m^{2}+8 m+8\right) z^{4 m}-2 m(m+3) \phi z^{2 m}+m \phi^{2}}{\eta_{1} \mu^{2} z^{4 m}-2 \eta_{2} \delta \mu \sigma^{m-1} z^{2 m}+m^{4} \delta^{2} \sigma^{2 m-2}}, & \text { for } Z M_{4}, \\ \eta_{1}{ }^{2 m-1} z^{m-1}{ }^{2 m} & \text { for } S M_{4},\end{cases}
$$

with $\delta=1+(1+m) z^{2}, \sigma=-1+(1+m)^{2} z^{2}, \rho=(m+2)^{2 m} z^{2 m} \mu, \lambda=m^{m}(m+$ $2)^{m} z^{2 m}, \mu=\left(\frac{m}{m+2}\right)^{m}, \phi=m^{1-m}(m+2)^{1-m} \delta \sigma^{m-1}, \eta_{1}=(m-2)(m+2)^{4 m+1}$, $\eta_{2}=(m+2)^{2 m-1}\left(m^{4}+2 m^{3}-2 m^{2}-4 m-8\right)$.

Since $H(z)$ in (5.3) or (5.4) defines a high-order rational function as the multiplicity $m$ increases, it is convenient to study the typical cases of $m \in\{2,3,4,5\}$ for locating the corresponding extraneous fixed points. In fact, Tables 1 and 2 list $H(z)$ and the extraneous fixed points $\xi$, respectively for the values of $2 \leq m \leq 5$. The numerator $F(z)$ or denominator $D(z)$ is shortened if its expression is so lengthy.

We further note that the stability of the extraneous fixed points of the listed methods in Table 2 varies in a variety of ways. The last three columns of Table 2 respectively indicate a number of attractive, indifferent, and repulsive extraneous fixed points. Interestingly, it is straightforward to show that the extraneous fixed points of Method $S_{4}$ are all found to be indifferent due to its inherent structure of $H(z)$. In the latter part of Section 7 complex dynamics will be discussed along with chaotic behavior of rational iterative maps (5.1) when applied to various polynomials $p(z)$, based on visual description of their 
Table 1. $A(z), F(z)$ and $D(z)$ of various fourth-order methods for typical cases

\begin{tabular}{|c|c|c|c|}
\hline Method & $m$ & $A(z)$ & $F(z)$ \\
\hline$O M_{4}$ & $\begin{array}{l}2 \\
3 \\
4 \\
5\end{array}$ & $\begin{array}{l}z / 8 \\
3 z \\
z \\
5 z\end{array}$ & $\begin{array}{c}\left(z^{2}-4 z-1\right)^{2}\left(1+16 z+156 z^{2}+\cdots+z^{8}\right) \\
169957+2185776 z+3231732 z^{2}+\cdots+25 z^{24} \\
21295541+494285440 z+\cdots-459 z^{32} \\
1821421328553+67008048468600 z+\cdots+2960433 z^{40}\end{array}$ \\
\hline$L M_{4}$ & $\begin{array}{l}2 \\
3 \\
4 \\
5\end{array}$ & $\begin{array}{l}z^{4} \\
3 \\
4 \\
5\end{array}$ & $\begin{array}{c}32 \\
-1+28 z^{2}-128 z^{4}+1001 z^{6} \\
1-70 z^{2}+1500 z^{4}-6250 z^{6}+32467 z^{8} \\
-3+414 z^{2}-20736 z^{4}+419904 z^{6}-1679616 z^{8}+7282537 z^{10}\end{array}$ \\
\hline $\mathrm{SSM}_{4}$ & $\begin{array}{l}2 \\
3 \\
4 \\
5\end{array}$ & $\begin{array}{l}2 \\
3 \\
4 \\
5\end{array}$ & $\begin{array}{c}\left(1-12 z^{2}+46 z^{4}-60 z^{6}+1049 z^{8}\right) \\
23-1288 z^{2}+23920 z^{4}+\cdots+11950373 z^{12} \\
7-980 z^{2}+55300 z^{4}+\cdots+8641095007 z^{16} \\
113-31188 z^{2}+3714084 z^{4}+\cdots+1145335160540383 z^{20}\end{array}$ \\
\hline$Z M_{4}$ & $\begin{array}{l}2 \\
3 \\
4 \\
5\end{array}$ & $\begin{array}{l}1 \\
1 \\
1 \\
1\end{array}$ & $\begin{array}{c}\left(1+2 z-4 z^{2}-2 z^{3}+19 z^{4}\right)\left(1-2 z-4 z^{2}+2 z^{3}+19 z^{4}\right) \\
1-56 z^{2}+1040 z^{4}+\cdots+190651 z^{12} \\
1-140 z^{2}+7900 z^{4}+\cdots+538664425 z^{16} \\
1-276 z^{2}+32868 z^{4}+\cdots+5065625942791 z^{20}\end{array}$ \\
\hline$S M_{4}$ & $\begin{array}{l}2 \\
3 \\
4 \\
5\end{array}$ & $\begin{array}{c}z^{4} \\
z^{6} \\
z^{8} \\
z^{10}\end{array}$ & $\begin{array}{c}32 \\
1200(-1+4 z)^{2}(1+4 z)^{2}\left(1+4 z^{2}\right) \\
13824(-1+5 z)^{3}(1+5 z)^{3}\left(1+5 z^{2}\right) \\
960400(-1+6 z)^{4}(1+6 z)^{4}\left(1+6 z^{2}\right)\end{array}$ \\
\hline & & & $D(z)$ \\
\hline$O M_{4}$ & $\begin{array}{l}2 \\
3 \\
4 \\
5\end{array}$ & $\begin{array}{l}z / 8 \\
3 z \\
z \\
5 z\end{array}$ & $\begin{array}{c}\left(z^{2}-2 z-1\right)\left(3+6 z-32 z^{2}+\cdots+6 z^{9}\right) \\
2\left(z^{2}-6 z-1\right)^{2}\left(z^{2}-3 z-1\right)\left(14161+97032 z+\cdots-675 z^{18}\right) \\
2 \tau_{1}\left(166375+2531064 z+\cdots+1593 z^{26}\right) \\
4 \tau_{2}\left(15178486401+406610605840 z+\cdots-19032727 z^{34}\right)\end{array}$ \\
\hline$L M_{4}$ & $\begin{array}{l}2 \\
3 \\
4 \\
5\end{array}$ & $\begin{array}{l}z^{4} \\
3 \\
4 \\
5\end{array}$ & $\begin{array}{c}11 z^{4}+6 z^{2}-1 \\
2\left(1-28 z^{2}+128 z^{4}+349 z^{6}\right) \\
-1+70 z^{2}-1500 z^{4}+6250 z^{6}+22829 z^{8} \\
2\left(1-138 z^{2}+6912 z^{4}-139968 z^{6}+559872 z^{8}+2574571 z^{10}\right)\end{array}$ \\
\hline$S S M_{4}$ & $\begin{array}{l}2 \\
3 \\
4 \\
5\end{array}$ & $\begin{array}{l}2 \\
3 \\
4 \\
5\end{array}$ & $\begin{array}{c}(-1+3 z)^{2}(1+3 z)^{2}\left(1+3 z^{2}\right)^{2} \\
8(-1+4 z)^{4}(1+4 z)^{4}\left(1+4 z^{2}\right)^{2} \\
(-1+5 z)^{6}(1+5 z)^{6}\left(1+5 z^{2}\right)^{2} \\
8(-1+6 z)^{8}(1+6 z)^{8}\left(1+6 z^{2}\right)^{2}\end{array}$ \\
\hline$Z M_{4}$ & $\begin{array}{l}2 \\
3 \\
4 \\
5\end{array}$ & $\begin{array}{l}1 \\
1 \\
1 \\
1\end{array}$ & $\begin{array}{c}128 z^{8} \\
45000 z^{12} \\
95551488 z^{16} \\
720600125000 z^{20}\end{array}$ \\
\hline$S M_{4}$ & $\begin{array}{l}2 \\
3 \\
4 \\
5\end{array}$ & $\begin{array}{l}z^{4} \\
z^{6} \\
z^{8} \\
z^{10}\end{array}$ & $\begin{array}{c}-1+6 z^{2}+11 z^{4} \\
3-168 z^{2}+3120 z^{4}+\cdots+288703 z^{12} \\
1-140 z^{2}+7900 z^{4}+\cdots+193295977 z^{16} \\
1-276 z^{2}+32868 z^{4}+\cdots+1400659421421 z^{20}\end{array}$ \\
\hline
\end{tabular}

We denote $\tau_{1}=\left(z^{2}-8 z-1\right)^{2}\left(z^{2}-4 z-1\right)$ and $\tau_{2}=\left(z^{2}-10 z-1\right)^{2}\left(z^{2}-5 z-1\right)$. 
Table 2. Extraneous fixed points $\xi$ with stability check for selected cases with $2 \leq m \leq 5$

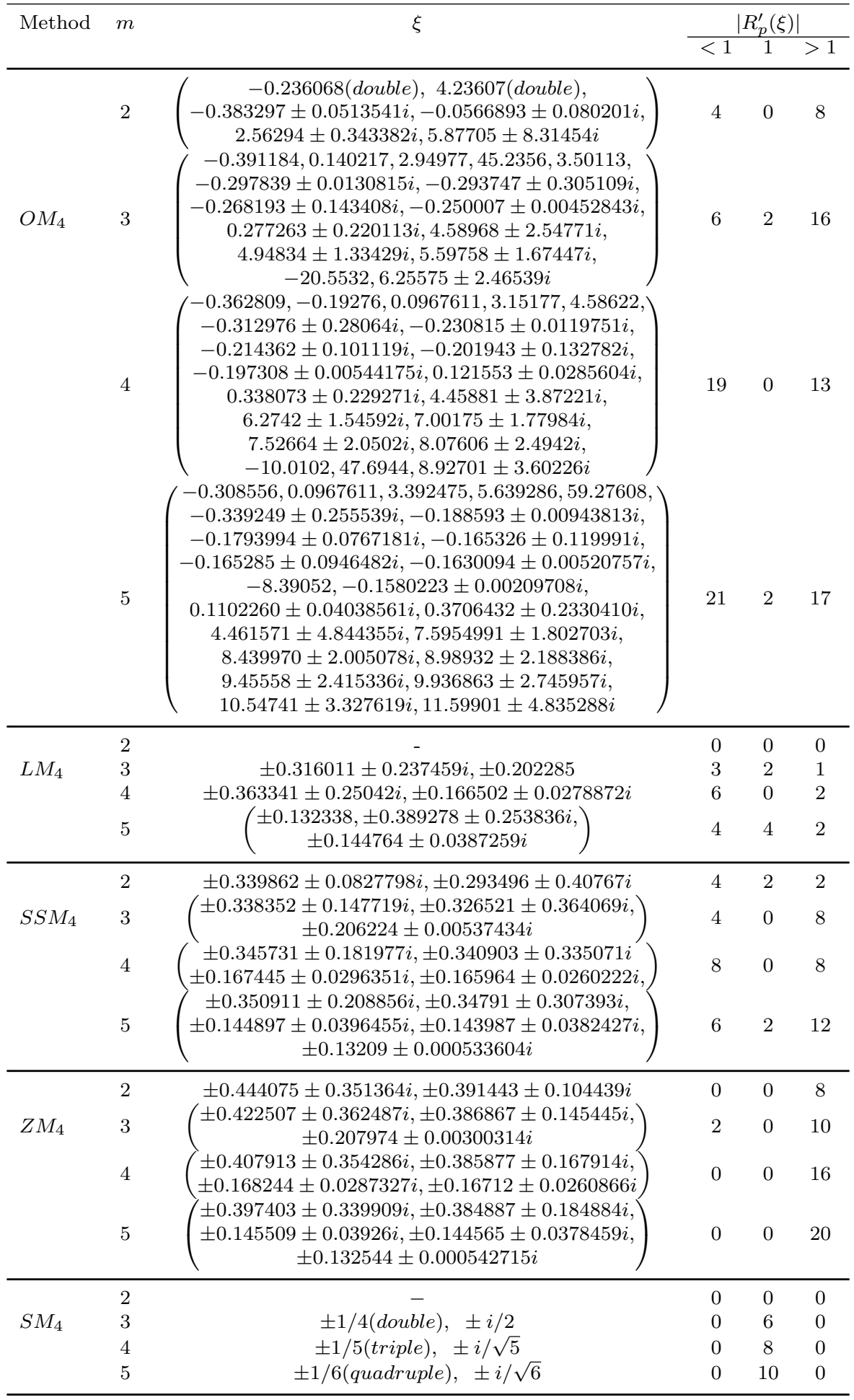


basins of attraction along with comparison of their dynamic properties and characteristics.

\section{$6 \quad$ Numerical illustrations}

In this section, we shall check the effectiveness of our proposed methods and validity of the theoretical results. We employ the present methods namely, method (2.6), (2.8), (2.9) and (3.1) (for $\gamma=1$ ) denoted by $M R M_{2}, M H M_{3}$, $M S M_{3}$ and $O M_{4}$ respectively to solve a variety of nonlinear equations which are mentioned in examples $1-3$.

First of all, we compare our one-point methods with one-point methods which are available in the literature namely, the method of Rall's method [12] $\left(R M_{2}\right)$, Halley's method [5] $\left(H M_{3}\right)$, super-Halley method [5] $\left(S M_{3}\right)$.

After that we compare our optimal method $O M_{4}$ with optimal methods proposed by Soleymani et al. [16], between them we will choose their best expression (18) which is denoted by $\left(S M_{4}\right)$, and also choose expression (11) proposed by Zhou et al. [19], denoted by $\left(Z M_{4}\right)$. In addition to this, we also compare our optimal method with the one which has been recently developed by Sharma and Sharma [15], denoted by $\left(S S M_{4}\right)$. Moreover, we compare $O M_{4}$ with the schemes given by Li et al. [8], out of which we have chosen method (75), denoted by $\left(L M_{4}\right)$.

Finally, we compare them with schemes proposed by Argyros et al. [1], out of them we consider expression (2.4) with $\left(H_{1}(\tau), \gamma=-0.01, \alpha=1\right)$ and $\left(H_{2}(\tau), \gamma=-0.01, \alpha=0\right)$, called by $A M_{1}$ and $A M_{2}$, respectively. Actually, the methods $A M_{1}$ and $A M_{2}$ are designed only for simple roots according to their paper. Hence we expect the convergence of simple-root finders $A M_{1}$ and $A M_{2}$ is of linear character when locating a multiple root. It is well-known that Newton's method of simple roots for multiple roots gives linear convergence. Such aspects of $A M_{1}$ and $A M_{2}$ are evidently indicated in Tables 3-5 with a larger number of iterations as well as relatively bigger errors for convergence than our proposed methods.

For better comparisons of our proposed methods, we have given three comparison tables in each example: one is corresponding to absolute error value of given nonlinear functions with the same total number of functional evaluations (TNFE $=12$ ); second one is with respect to number of iterations taken by each method to obtain the accuracy of root up to 35 significant digits and the last one is regarding computational order of convergence. TNFE in the case of modified Newton's method, it will consume 12 functional evaluations in 6 iterations because it takes 2 functional evaluations per full iteration on the other hand, third-order methods namely, $\mathrm{HM}_{3}, \mathrm{SM}_{3}, M H M_{3}$ and $M S M_{3}$ will consume 12 functional evaluations in 4 iterations because they required three functional evaluations per iteration, and same law for fourth-order methods. This means the absolute error in the function after consuming 12 functional evaluations presented in the Tables 1-3 for second, third and fourth order methods is $\left|f\left(x_{6}\right)\right|$, $\left|f\left(x_{4}\right)\right|$ and $\left|f\left(x_{4}\right)\right|$, respectively.

Further, we use the following formula to calculate the computational order 
of convergence (see [18])

$$
\rho=\ln \left|\left(x_{n+1}-r_{m}\right) /\left(x_{n}-r_{m}\right)\right| / \ln \left|\left(x_{n}-r_{m}\right) /\left(x_{n-1}-r_{m}\right)\right|, \quad n>1 .
$$

All the computations have been performed using the programming package Mathematica 9 with multiple precision arithmetic. We use $\epsilon=10^{-100}$ as a tolerance error and $\mathrm{A}-h$ (or $\mathrm{A}+h$ ) stands for $A \times 10^{-h}\left(\right.$ or $A \times 10^{+h}$ ). The following stopping criteria are used for computer programs:

(i) $\left|x_{n+1}-x_{n}\right|<\epsilon$ and $(i i)\left|f\left(x_{n+1}\right)\right|<\epsilon$.

Example 1. Consider the following $8 \times 8$ matrix

$$
B=\left[\begin{array}{cccccccc}
5 & 8 & 0 & 2 & 6 & -6 & 0 & -1 \\
0 & 1 & 0 & 0 & 0 & 0 & 0 & 0 \\
6 & 18 & -1 & 1 & 13 & -9 & 0 & 0 \\
3 & 6 & 0 & 4 & 6 & -6 & 0 & 0 \\
4 & -14 & -2 & 0 & 11 & -6 & 0 & 0 \\
6 & 18 & -2 & 1 & 13 & -8 & 0 & 0 \\
0 & 0 & 0 & 0 & 0 & 0 & 0 & -1 \\
0 & 0 & 0 & 0 & 0 & 0 & 0 & -19
\end{array}\right]
$$

The corresponding characteristic polynomial of this matrix is as follows:

$$
f_{1}(x)=x(x-1)^{3}(x-2)(x-3)(x-4)(x+19) .
$$

The above equation has one multiple root at $x=1$ of multiplicity three. It can be seen that Newton's method and its variants do not necessarily converge to the required root that is nearest to the starting value. For example, the methods $R M_{2}, Z M_{4}, S S M_{4}$ and $L M_{4}$ with initial guess $x_{0}=0.3$ diverge from the required root. In addition, the method $S S M_{4}$ also divergent for the other initial guesses 1.5 and 1.8. However, newly proposed families of methods namely, (2.6), (2.8), (2.9) and (3.1) (for $\gamma=1$ ) do not exhibit this type of behavior.

Example 2. Let us consider the another nonlinear equation, which is given by

$$
f_{2}(x)=x^{2} \sin 4 x
$$

This function has an infinite number of zeros but our desired root is $r=0$ of multiplicity three, which is correct up to 35 digits. It can be seen that Newton's method and it's variants do not necessarily converge to the root that is nearest to the starting value. For example, methods $S S M_{4}$ and $L M_{4}$ are divergent for the initial guesses -0.4 and 0.4 . However, newly proposed families of methods namely, (2.6), (2.8), (2.9) and (3.1) (for $\gamma=1$ ) do not exhibit this type of behavior.

Example 3. Consider the following nonlinear equation

$$
f_{3}(x)=\left(e^{-x}+\sin x\right)^{3} .
$$

We find that this function has a finite number of zeros but our desired root is given by $r=3.1830630119333635919391869956363946$ of multiplicity three. It can 
Table 3. Test problem (6.1)

\begin{tabular}{|c|c|c|c|c|c|c|c|c|}
\hline$f(x)$ & $x_{0}$ & $R M_{2}$ & $M R M_{2}$ & $H M_{3}$ & $\mathrm{MHM}_{3}$ & $S M_{3}$ & $M S M_{3}$ & $S M_{4}$ \\
\hline \multicolumn{9}{|c|}{ Comparison of different iterative methods with the same $\mathrm{TNFE}=12$} \\
\hline \multirow{4}{*}{$f_{1}(x)$} & 0.3 & $D$ & $1.3(-136)$ & $2.6(-33)$ & $1.3(-53)$ & $4.3(-113)$ & $8.3(-264)$ & $3(-76)$ \\
\hline & 0.4 & $9.1(-108)$ & $2.1(-145)$ & $8.0(-66)$ & $1.7(-70)$ & $3.7(-106)$ & $7.6(-101)$ & $3(-68)$ \\
\hline & 1.5 & $6.2(-163)$ & $7.2(-140)$ & $8.5(-75)$ & $1.3(-82)$ & $6.0(-157)$ & $6.0(-208)$ & $8.1(-4)$ \\
\hline & 1.8 & $3.1(-15)$ & $3.3(-39)$ & $1.3(-1)$ & $4.6(-24)$ & $3.4(-10)$ & $2.5(-67)$ & $9.3(+0)$ \\
\hline \multicolumn{9}{|c|}{ Comparison of different iterative methods with respect to number of iterations } \\
\hline \multirow{4}{*}{$f_{1}(x)$} & 0.3 & $\mathrm{D}$ & 8 & 6 & 6 & 5 & 5 & 5 \\
\hline & 0.4 & 8 & 8 & 6 & 6 & 5 & 5 & 6 \\
\hline & 1.5 & 8 & 8 & 6 & 6 & 5 & 5 & 947 \\
\hline & 1.8 & 11 & 9 & 332 & 7 & 7 & 6 & 892 \\
\hline \multicolumn{9}{|c|}{ Computational order of convergence of different iterative methods } \\
\hline \multirow{5}{*}{$f_{1}(x)$} & 0.3 & $\mathrm{D}$ & 2.0000 & 3.0000 & 3.0000 & 3.0000 & 3.0000 & 4.0000 \\
\hline & 0.4 & 2.0000 & 2.0000 & 3.0000 & 3.0000 & 3.0000 & 3.0000 & 4.0000 \\
\hline & 1.5 & 2.0000 & 2.0000 & 3.0000 & 3.0000 & 3.0000 & 3.0000 & 1.0000 \\
\hline & 1.8 & 2.0000 & 2.0000 & 1.0000 & 3.0000 & 3.0000 & 3.0000 & 1.0000 \\
\hline & & $Z M_{4}$ & $\mathrm{SSM}_{4}$ & $L M_{4}$ & $A M_{1}$ & $A M_{2}$ & $O M_{4}$ & \\
\hline
\end{tabular}

Comparison of different iterative methods with the same $\mathrm{TNFE}=12$

$\begin{array}{rlllllll} & 0.3 & \mathrm{D} & \mathrm{D} & \mathrm{D} & 5.0(-3) & 1.4(-9) & 6.2(-84) \\ f_{1}(x) & 0.4 & 1.8(-68) & 6.2(-68) & 1.5(-67) & 2.1(-8) & 5.0(-8) & 3.4(-151) \\ & 1.5 & 7.9(+59) & \mathrm{D} & 2.1(-16) & 1.9(-3) & 4.3(-3) & 3.6(-492) \\ & 1.8 & 1.7(+21) & \mathrm{D} & 1.5(-252) & 9.1(-25)^{*} & 2.6(-107)^{*} & 3.0(-213)\end{array}$

Comparison of different iterative methods with respect to number of iterations

$\begin{array}{llllllll} & 0.3 & \mathrm{D} & \mathrm{D} & \mathrm{D} & 265 & 6 & 6 \\ f_{1}(x) & 0.4 & 6 & 6 & 6 & 261 & 294 & 5 \\ & 1.5 & 33 & \mathrm{D} & 7 & 265 & 298 & 4 \\ & 1.8 & 16 & \mathrm{D} & 5 & 4^{*} & 4 & 5\end{array}$

Computational order of convergence of different iterative methods

$\begin{array}{rlllllll}f_{1}(x) & 0.3 & \mathrm{D} & \mathrm{D} & \mathrm{D} & 1.0000 & 4.0000 & 4.0000 \\ & 0.4 & 4.0000 & 4.0000 & 4.0000 & 1.0000 & 1.0000 & 3.9996 \\ & 1.5 & 4.0000 & \mathrm{D} & 4.0000 & 1.0000 & 1.0000 & 4.0000 \\ & 1.8 & 4.0000 & \mathrm{D} & 4.0000 & 4.0000^{*} & 4.0000^{*} & 4.0000\end{array}$

TNFE=total number of functional evaluations. $\quad$ D: stands for divergence. * : stands for converge to an undesired root. 
Table 4. Test Problem (6.2)

\begin{tabular}{|c|c|c|c|c|c|c|c|c|}
\hline$f(x)$ & $x_{0}$ & $R M_{2}$ & $M R M_{2}$ & $H M_{3}$ & $\mathrm{MHM}_{3}$ & $S M_{3}$ & $M S M_{3}$ & $\mathrm{SM}_{4}$ \\
\hline \multicolumn{9}{|c|}{ Comparison of different iterative methods with the same TNFE $=12$} \\
\hline \multirow{2}{*}{$f_{2}(x)$} & -0.4 & $4.6(-382)$ & $4.6(-466)$ & $4.0(-72)$ & $3.7(-75)$ & $1.0(-175)$ & $1.6(-153)$ & $6.9(-13)$ \\
\hline & 0.4 & $4.6(-382)$ & $4.6(-466)$ & $4.0(-72)$ & $3.7(-75)$ & $1.0(-175)$ & $1.6(-153)$ & $6.9(-13)$ \\
\hline \multicolumn{9}{|c|}{ Comparison of different iterative methods with respect to number of iterations } \\
\hline \multirow{2}{*}{$f_{2}(x)$} & -0.4 & 6 & 6 & 6 & 6 & 5 & 5 & 6 \\
\hline & 0.4 & 6 & 6 & 6 & 6 & 5 & 5 & 7 \\
\hline \multicolumn{9}{|c|}{ Computational order of convergence of different iterative methods } \\
\hline \multirow{3}{*}{$f_{2}(x)$} & -0.4 & 3.0000 & 3.0000 & 3.0000 & 3.0000 & 3.0000 & 3.0000 & 5.0000 \\
\hline & 0.4 & 3.0000 & 3.0000 & 3.0000 & 3.0000 & 3.0000 & 3.0000 & 5.0000 \\
\hline & & $Z M_{4}$ & $\mathrm{SSM}_{4}$ & $L M_{4}$ & $A M_{1}$ & $A M_{2}$ & $O M_{4}$ & \\
\hline \multicolumn{9}{|c|}{ Comparison of different iterative methods with the same TNFE $=12$} \\
\hline \multirow{2}{*}{$f_{2}(x)$} & -0.4 & $1.8(-10)$ & $\mathrm{D}$ & $\mathrm{D}$ & $5.0(-6)$ & $1.2(-4)$ & $3.4(-185)$ & \\
\hline & 0.4 & $1.8(-10)$ & $\mathrm{D}$ & $\mathrm{D}$ & $5.0(-6)$ & $1.2(-4)$ & $3.4(-185)$ & \\
\hline \multicolumn{9}{|c|}{ Comparison of different iterative methods with respect to number of iterations } \\
\hline \multirow{2}{*}{$f_{2}(x)$} & -0.4 & 7 & $\mathrm{D}$ & $\mathrm{D}$ & 261 & 265 & 5 & \\
\hline & 0.4 & 7 & $\mathrm{D}$ & $\mathrm{D}$ & 261 & 265 & 5 & \\
\hline \multicolumn{9}{|c|}{ Computational order of convergence of different iterative methods } \\
\hline \multirow{2}{*}{$f_{2}(x)$} & -0.4 & 5.000 & $\mathrm{D}$ & $\mathrm{D}$ & 1.0000 & 1.0000 & 5.0000 & \\
\hline & 0.4 & 5.000 & $\mathrm{D}$ & $\mathrm{D}$ & 1.0000 & 1.0000 & 5.0000 & \\
\hline
\end{tabular}

be seen that Newton's method and it's variants do not necessarily converge to the root that is nearest to the starting value. For example, method $S S M_{4}$ is divergent for initial guess $x_{0}=2$, while $S M_{4}, Z M_{4}$ and $L M_{4}$ converge to the undesired root. Similarly, methods $S M_{4}$ and $S S M_{4}$ are divergent for initial guess $x_{0}=4.2$ while $Z M_{4}$ converges to the undesired root. However, newly proposed families of methods namely, (2.6), (2.8), (2.9) and (3.1) (for $\gamma=1$ ) do not exhibit this type of behavior.

\section{Basins of attraction}

This section directly describes the dynamics of iterative map (5.2) based on visual display of their basins of attraction when $f(z)$ is applied to a complex polynomial $p(z)$. We here investigate the comparison of basins of attraction for the attained best five or six multiple-root finders. It is known that the corresponding fractal of an iterative root-finding method is a boundary set in the complex plane, which is characterized by an iterative method applied to a fixed polynomial $p(z) \in \mathbb{C}$, see e.g. $[9,14]$. The aim herein is to use basins of attraction as another way for comparing the convergence of the iteration algorithms in a global sense.

From the dynamical point of view, we consider a rectangle $D=[-3,3] \times$ $[-3,3] \in \mathbb{C}$ with a $400 \times 400$ grid, and we assign a color to each point $z_{0} \in D$ according to the multiple root at which the corresponding iterative method starting from $z_{0}$ converges, and we mark the point as black if the method does not converge. In this section, we consider the stopping criterion for convergence to be less than $10^{-4}$ wherein the maximum number of full cycles for each 
Table 5. Test problem (6.3)

\begin{tabular}{|c|c|c|c|c|c|c|c|}
\hline$f(x)$ & $x_{0} \quad R M_{2}$ & $M R M_{2}$ & $H M_{3}$ & $M H M_{3}$ & $S M_{3}$ & $M S M_{3}$ & $\mathrm{SM}_{4}$ \\
\hline \multicolumn{8}{|c|}{ Comparison of different iterative methods with the same $\mathrm{TNFE}=12$} \\
\hline \multirow{2}{*}{$f_{3}(x)$} & $2.06 .5(-112)$ & $8.1(-147)$ & $2.3(-67)$ & $4.2(-75)$ & $3.1(-165)$ & $2.1(-124)$ & $9.0(-10)^{*}$ \\
\hline & $4.21 .3(-109$ & $5.7(-177)$ & $5.5(-69)$ & $6.6(-76)$ & $2.5(-142)$ & $8.2(-151)$ & 224 \\
\hline \multicolumn{8}{|c|}{ Comparison of different iterative methods with respect to number of iterations } \\
\hline \multirow{2}{*}{$f_{3}(x)$} & 2.08 & 8 & 6 & 6 & 5 & 5 & $7^{*}$ \\
\hline & 4.28 & 8 & 6 & 6 & 5 & 5 & $\mathrm{D}$ \\
\hline \multicolumn{8}{|c|}{ Computational order of convergence of different iterative methods } \\
\hline \multirow{3}{*}{$f_{3}(x)$} & 2.02 .0000 & 2.0000 & 3.0000 & 3.0000 & 3.0000 & 3.0000 & $4.0000^{*}$ \\
\hline & 4.22 .0000 & 2.0000 & 3.0000 & 3.0000 & 3.0000 & 3.0000 & $\mathrm{D}$ \\
\hline & $Z M_{4}$ & $S_{S} M_{4}$ & $L_{4}$ & $A M_{1}$ & $A M_{2}$ & $\mathrm{OM}_{4}$ & \\
\hline \multicolumn{8}{|c|}{ Comparison of different iterative methods with the same $\mathrm{TNFE}=12$} \\
\hline \multirow{2}{*}{$f_{3}(x)$} & $2.01 .8(-30)^{*}$ & $2.3(+5581)^{*}$ & $9.0(+55)^{*}$ & $3.4(-4)$ & $2.0(-5)$ & $1.8(-446)$ & \\
\hline & $4.2 \mathrm{D}$ & $\mathrm{D}$ & $8.7(-84)$ & $1.8(-5)$ & $1.1(-5)$ & $8.0(-232)$ & \\
\hline \multicolumn{8}{|c|}{ Comparison of different iterative methods with respect to number of iterations } \\
\hline \multirow{2}{*}{$f_{3}(x)$} & $2.05^{*}$ & 2224 & 32 & 266 & 249 & 4 & \\
\hline & $4.2 \mathrm{D}$ & $\mathrm{D}$ & 5 & 266 & 249 & 5 & \\
\hline \multicolumn{8}{|c|}{ Computational order of convergence of different iterative methods } \\
\hline \multirow{2}{*}{$f_{3}(x)$} & $2.04 .2482^{*}$ & 1.4735 & 5.0000 & 1.0000 & 1.0000 & 3.7333 & \\
\hline & $4.2 \mathrm{D}$ & $\mathrm{D}$ & 4.0000 & 1.0000 & 1.0000 & 4.0000 & \\
\hline
\end{tabular}

*: stands for convergence to an undesired root.

method is considered to be 100. In this way, we distinguish the attraction basins by their colors for different methods. The lighter colour of basin of attraction means faster convergence to the required root, while darker one means slower convergence.

For the first two test problems, we have taken the polynomial functions.

Problem 1. Let $p_{1}(z)=\left(z^{5}+2 z-1\right)^{3}$, having multiple zeros $\{-0.945068$ $\pm 0.854518 i, 0.701874 \pm 0.879697 i, 0.486389\}$ with multiplicity three. It is straight forward to see from Figure 1 that our methods, namely $M R M_{2}$, $\mathrm{MHM}_{3}$ and $\mathrm{MSM}_{3}$ have the same basin of attraction as compared to the classical $R M_{2}, H M_{3}$ and $S M_{3}$, respectively. Further, we observe from Figure 2 that our method $\mathrm{OM}_{4}$ has lesser number of divergent points in comparison to the methods namely, $S M_{4}, Z M_{4}$ and $L M_{4}$ and shows less chaotic behavior as compared to methods namely, $S M_{4}, Z M_{4}, L M_{4}$ and $S S M_{4}$ in the above mentioned region.

Problem 2. Let $p_{2}(z)=\left(z^{4}+z\right)^{3}$, having multiple zeros $\{-1,0.5+0.866025 i$, $0.5-0.866025 i, 0\}$ with multiplicity three. We can easily say, after seeing the Figure 3 that our methods, namely $M R M_{2}$ and $\mathrm{MHM}_{3}$ have the same basin of attraction as compared to the classical $R M_{2}$ and $H M_{3}$, respectively. Further, from Figure 4, we observe that our method $O M_{4}$ has lesser number of divergent points in comparison to the methods namely, $S M_{4}, Z M_{4}$ and $L M_{4}$, lighter and lager basins of attraction belong to our method $O M_{4}$ as compared to other methods namely, $S M_{4}, Z M_{4}, L_{4}$ and $S S M_{4}$. 

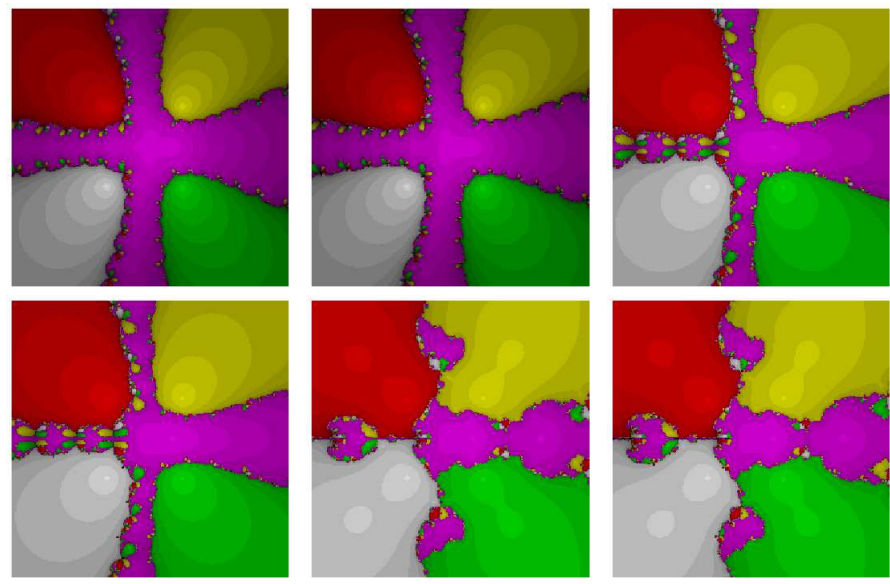

Figure 1. The basins of attraction for $R M_{2}, M R M_{2}, H M_{3}, M H M_{3}, S M_{3}$ and $M S M_{3}$, from left to right and top to bottom, respectively in Problem 1.


Figure 2. The basins of attraction for $S M_{4}, Z M_{4}, S S M_{4}, L M_{4}$ and $O M_{4}$, from left to right and top to bottom, respectively in Problem 1. 

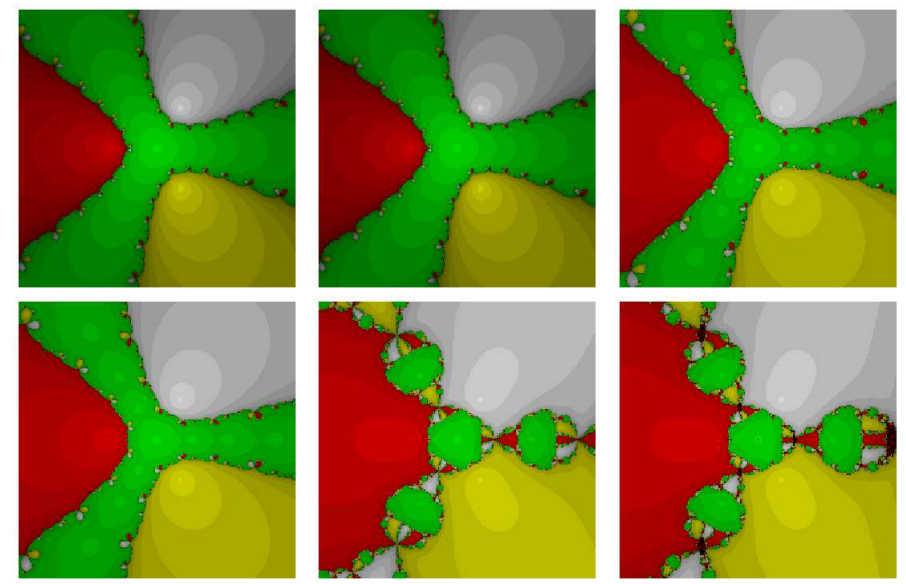

Figure 3. The basins of attraction for $R M_{2}, M R M_{2}, H M_{3}, M H M_{3}, S M_{3}$ and $M S M_{3}$, from left to right and top to bottom, respectively in Problem 2.
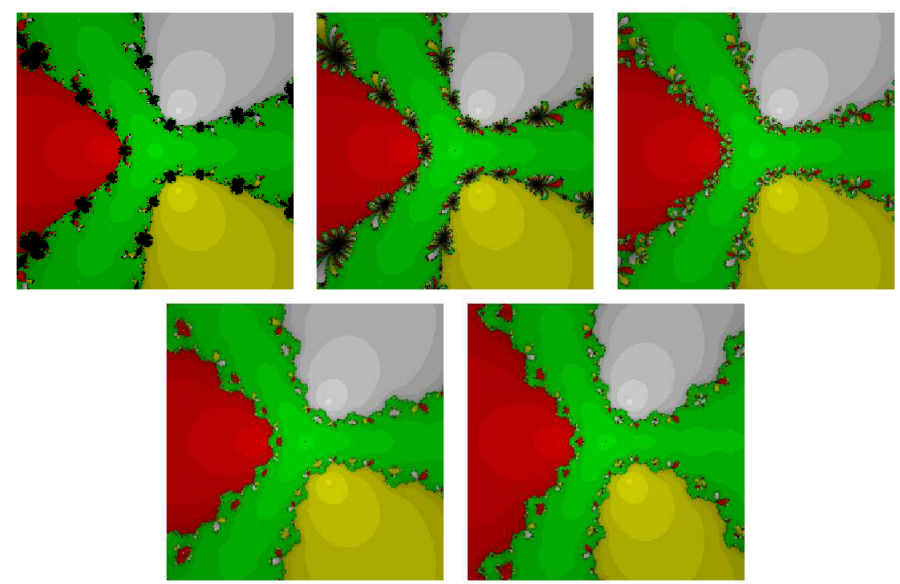

Figure 4. The basins of attraction for $S M_{4}, Z M_{4}, S S M_{4}, L M_{4}$ and $O M_{4}$, from left to right and top to bottom, respectively in Problem 2. 
The last test problem is a non-polynomial function as follows.

Problem 3. Let $p_{3}(z)=\left(z^{6}+\frac{1}{z}\right)^{4}$, having multiple zeros $\{-0.62349 \pm 0.781831 i$, $0.222521 \pm 0.974928 i,-1,0.900969 \pm 0.433884 i\}$ with multiplicity four. Figure 5 demonstrates that our methods, namely $\mathrm{MRM}_{2}, \mathrm{MHM}_{3}$ and $M \mathrm{MM}_{3}$ have the same basin of attraction as compared to the classical $R M_{2}, H M_{3}$ and $S M_{3}$, respectively. From Figure 6, we conclude that our method $O M_{4}$ has lesser number of divergent points in comparison to the methods namely, $S M_{4}$, $Z M_{4}$ and $L M_{4}$, and almost the same basin of attraction as compared to $S S M_{4}$ and $\mathrm{LM}_{4}$.
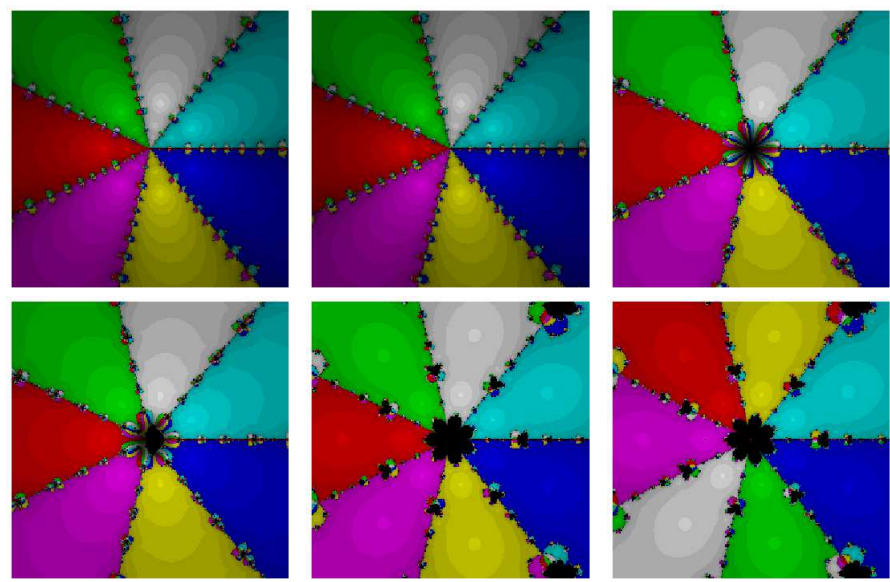

Figure 5. The basins of attraction for $R M_{2}, M R M_{2}, H M_{3}, M H M_{3}, S M_{3}$ and $M S M_{3}$, from left to right and top to bottom, respectively in Problem 3.
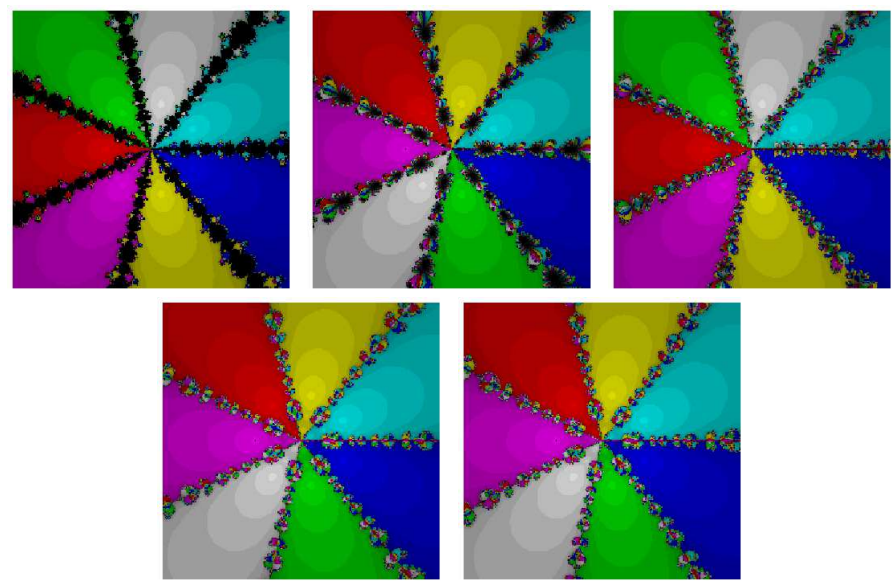

Figure 6. The basins of attraction for $S M_{4}, Z M_{4}, S S M_{4}, L M_{4}$ and $O M_{4}$, from left to right and top to bottom, respectively in Problem 3. 
On the other hand, the second part deals with the dynamics of selected methods $\mathrm{OM}_{4}, \mathrm{LM}_{4}, S S M_{4}, Z M_{4}$ and $S M_{4}$ behind the extraneous fixed points $\xi$ found from the roots of $H(z)$ whose construction is made by applying a simple quadratic polynomial $\left(z^{2}-1\right)^{m}$ to $f(z)$ in $H_{f}\left(x_{n}\right)$, using the rational iterative map (5.1). As mentioned in Section 5 about possible altering basins of attraction, we expect that the basins of attraction associated with extraneous fixed points would affect the Julia set basin boundaries. To visualize such basins of attraction with altered Julia set boundaries for specific examples, we employ four more examples described below in Problems 4-7. Indeed, Figures 7-10 well illustrate the affected Julia set boundaries.

Problem 4. Let $p_{4}(z)=\left(z^{2}-1\right)^{2}$, having multiple zeros $\{-1,1\}$ with multiplicity two. From Figure 7, we conclude that our method $O M_{4}$ has larger and brighter basins of attraction to the root -1 as compared to the methods namely, $L M_{4}, S S M_{4}, Z M_{4}$ and $S M_{4}$.

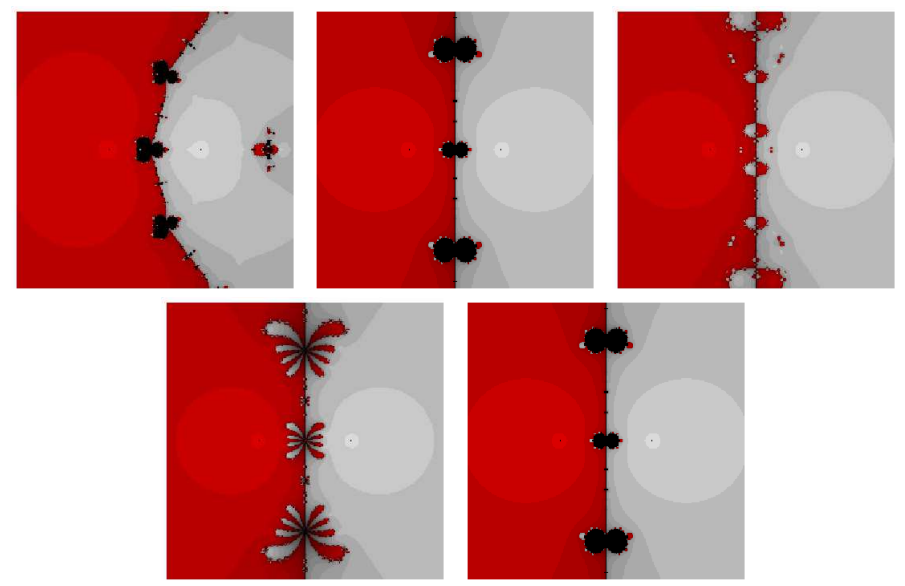

Figure 7. The basins of attraction for $O M_{4}, L M_{4}, S S M_{4}, Z M_{4}$ and $S M_{4}$, from left to right and top to bottom, respectively in Problem 4.

Problem 5. Let $p_{5}(z)=\left(z^{2}-1\right)^{3}$, having multiple zeros $\{-1,1\}$ with multiplicity three. Figure 8 demonstrate that our method $O M_{4}$ has larger and brighter basins of attraction to the root -1 as compared to the methods namely, $\mathrm{LM}_{4}, \mathrm{SSM}_{4}, Z_{4} M_{4}$ and $\mathrm{SM}_{4}$. Further, our method have very few divergent points in this particular region while $S M_{4}$ has many divergent points.

Problem 6. Let $p_{6}(z)=\left(z^{3}-z\right)^{4}$, having multiple zeros $\{-1,1,0\}$ with multiplicity four. If we inspect the basins of "0" more closely, $S M_{4}$ has the largest basin containing a huge number of divergent points and $Z M_{4}$ a medium basin containing a significant number of chaotic as well as divergent points. On the other hand, $\mathrm{OM}_{4}, \mathrm{LM}_{4}$ and $S S M_{4}$ has smaller basins containing less chaotic and less divergent points. It is, overall, straightforward in view of Figure 9 that 

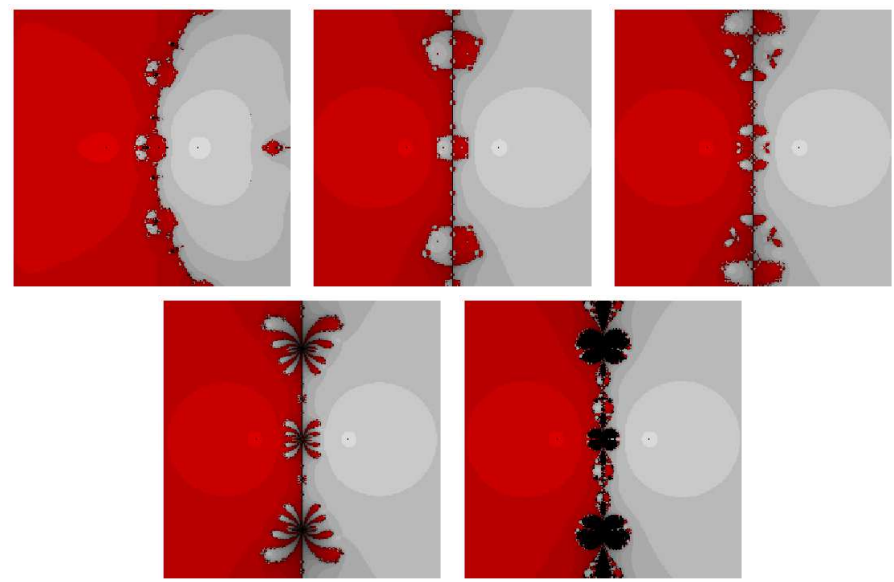

Figure 8. The basins of attraction for $O M_{4}, L M_{4}, S S M_{4}, Z M_{4}$ and $S M_{4}$, from left to right and top to bottom, respectively in Problem 5.

our method $\mathrm{OM}_{4}$ has lesser chaotic behavior and larger basins of attraction as compared to other methods namely, $L M_{4}, S S M_{4}, Z M_{4}$ and $S M_{4}$. Further, our method has no divergent point in this particular region while $S M_{4}$ has many divergent points.
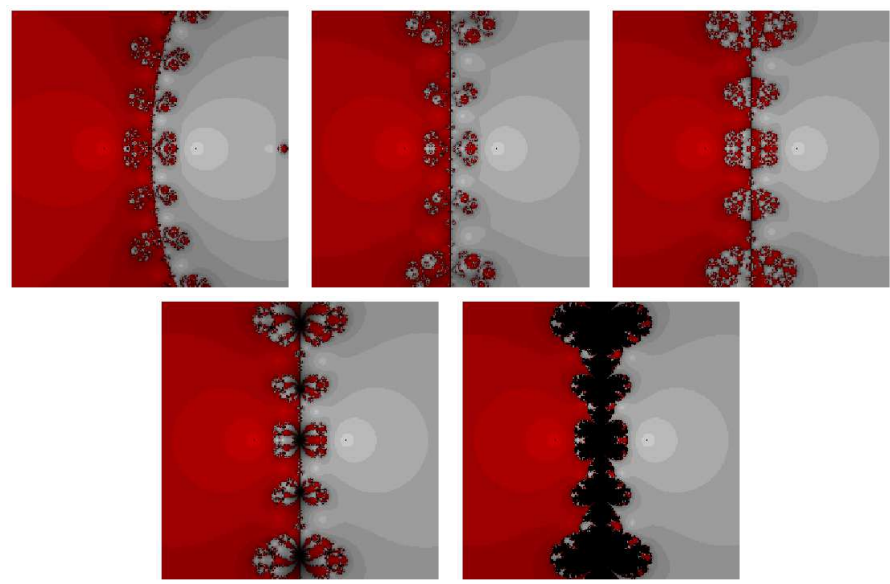

Figure 9. The basins of attraction for $O M_{4}, L M_{4}, S S M_{4}, Z M_{4}$ and $S M_{4}$, from left to right and top to bottom, respectively in Problem 6.

Problem 7. Let $p_{7}(z)=\left(z^{4}-1\right)^{5}$, having multiple zeros $\{ \pm i, \pm 1\}$ with multiplicity five. By closely looking the basin colors of attractors $\pm i$, we find that $\mathrm{SM}_{4}$ contains a large number of divergent points, while the rest of the listed methods contain much less divergent points. Overall, Figure 10 demonstrates that our method $\mathrm{OM}_{4}$ has lesser number of divergent points in comparison to 
the methods namely, $L M_{4}, S S M_{4}, Z M_{4}$ and $S M_{4}$. Further, our method also shows lesser chaotic behavior in this figure as compared to other mentioned methods.


Figure 10. The basins of attraction for $O M_{4}, L M_{4}, S S M_{4}, Z M_{4}$ and $S M_{4}$, from left to right and top to bottom, respectively in Problem 7 .

\section{Conclusions}

In this paper, we have proposed several formulas of second, third and fourthorder(optimal) methods respectively, for obtaining multiple roots of nonlinear equations numerically for the first time, which will converge to the required root even though the guess is far away from the desired root or the derivative is small in the vicinity of the root. Further, our proposed families of methods namely, (2.6), (2.8) and (2.9) have same error equations as those of classical Rall's method, super-Halley and Halley's method respectively, for multiple roots. These proposed methods offer a particular advantage for the cases where the traditional modified Newton's method and its variants of various order may not converge. Finally, we conclude that these methods are very effective in multi-precision environment and converge to a required root in a stable manner without divergence, oscillation or jumping problems. The dynamic study of the methods also supports the underlying theoretical aspects.

\section{References}

[1] I.K. Argyros, M. Kansal, V. Kanwar and S. Bajaj. Higher-order derivativefree families of Chebyshev-Halley type methods with or without memory for solving nonlinear equations. Appl. Math. Comput., 315:224-245, 2017. https://doi.org/10.1016/j.amc.2017.07.051.

[2] A. Cayley. Application of the Newton-Fourier method to an imaginary root of an equation. Quart. J. Pure Appl. Math., 16:179-185, 1879. 
[3] B. Kalantari and Y. Jin. On extraneous fixed-points of the basic family of iteration functions. BIT Numer. Math., 43(2):453-458, 2003. https://doi.org/10.1023/A:1026095904985.

[4] V. Kanwar, S. Bhatia and M. Kansal. New optimal class of higher-order methods for multiple roots, permitting $f^{\prime}\left(x_{n}\right)=0$. Appl. Math. Comput., 222(1):564-574, 2013. https://doi.org/10.1016/j.amc.2013.06.097.

[5] S. Kumar, V. Kanwar and S. Singh. On some modified families of multipoint iterative methods for multiple roots of nonlinear equations. Appl. Math. Comput., 218(14):7382-7394, 2012. https://doi.org/10.1016/j.amc.2011.12.081.

[6] H.T. Kung and J.F. Traub. Optimal order of one-point and multi-point iteration. Journal of the ACM, 21(4):643-651, 1974. https://doi.org/10.1145/321850.321860.

[7] S. Li, X. Liao and L. Cheng. A new fourth-order iterative method for finding multiple roots of nonlinear equations. Appl. Math. Comput., 215(3):1288-1292, 2009. https://doi.org/10.1016/j.amc.2009.06.065.

[8] S.G. Li, L.Z. Cheng and B. Neta. Some fourth-order nonlinear solvers with closed formulae for multiple roots. Comput. Math. Appl., 59(1):126-135, 2010. https://doi.org/10.1016/j.camwa.2009.08.066.

[9] B. Neta, M. Scot and C. Chun. Basins of attraction for several methods to find simple roots of nonnlinear equations. Appl. Math. Comput., 218(21):1054810556, 2012. https://doi.org/10.1016/j.amc.2012.04.017.

[10] A.M. Ostrowski. Solutions of Equations and System of Equations. Academic Press, New York, 1960.

[11] M.S. Petković, B. Neta, L.D. Petković and J. Džunić. Multipoint methods for solving nonlinear equations. Academic Press, 2012.

[12] L.B. Rall. Convergence of Newton's process to multiple solutions. Numer. Math., 9(1):23-37, 1966. https://doi.org/10.1007/BF02165226.

[13] E. Schröder. Uber unendlichviele algorithm zur auffosung der gleichungen. Math. Ann., 2(2):317-365, 1870. https://doi.org/10.1007/BF01444024.

[14] M. Scott, B. Neta and C. Chun. Basins attractors for various methods. Appl. Math. Comput., 218(6):2584-2599, 2011. https://doi.org/10.1016/j.amc.2011.07.076.

[15] J.R. Sharma and R. Sharma. Modified Jarratt method for computing multiple roots. Appl. Math. Comput., 217(2):878-881, 2010. https://doi.org/10.1016/j.amc.2010.06.031.

[16] F. Soleymani, D.K.R. Babajee and T. Lotfi. On a numerical technique for finding multiple zeros and its dynamic. J. Egypt. Math. Soc., 21(3):346-353, 2013. https://doi.org/10.1016/j.joems.2013.03.011.

[17] E.R. Vrscay and W.J. Gilbert. Extraneous fixed points, basin boundaries and chaotic dynamics for Shröder and König rational iteration functions. Numer. Math., 52(1):1-16, 1988. https://doi.org/10.1007/BF01401018.

[18] S. Weerakoon and T.G.I. Fernando. A variant of Newton's method with accelerated third-order convergence. Appl. Math. Lett., 13(8):87-93, 2000. https://doi.org/10.1016/S0893-9659(00)00100-2.

[19] X. Zhou, X. Chen and Y. Song. Constructing higher-order methods for obtaining the muliplte roots of nonlinear equations. J. Comput. Appl. Math., 235(14):4199-4206, 2011. https://doi.org/10.1016/j.cam.2011.03.014. 